\title{
MIPARTM: 2D and 3D Image Analysis Software Designed by Materials Scientists, for All Scientists
}

\author{
J.M. Sosa ${ }^{1}$, D.E. Huber ${ }^{1}$, B.A. Welk ${ }^{1}$ H.L. Fraser $^{1}$ \\ 1. Center for the Accelerated Maturation of Materials, Department of Materials Science and Engineering, \\ The Ohio State University, 1305 Kinnear Rd., Columbus, OH 43212
}

Many software programs have been developed independently for 2D and 3D image analysis, both commercial and open source. However, few, if any, can equip users with extensive toolsets for 2D and 3D materials characterization in a single package. To this end, Materials Image Processing and Automated Reconstruction (MIPAR) has been developed. MIPAR is based on an app-suite construction. The apps were designed as standalone programs, each suited for different tasks, but each capable of communication with the others. This paper will discuss the capabilities and purpose of each of these salient applications, as well as describe MIPAR's typical 2D and 3D characterization workflows.

Nearly all analysis efforts will originate in the first application known as the Image Processor (see Figure 1). This powerful, yet user-friendly environment allows users to develop a sequence of processing steps known as a recipe. The specific steps and parameters constituting a recipe will vary based on the user's intent, but the ultimate goal of most recipes is the same: to segment raw intensity into a set of features. A user's process and parameter selections are recorded real-time, permitting subsequent parameter editing as well as process removal or insertion.

Following completion of a recipe, it may be saved and loaded into the second app, the Batch Processor (see Figure 2). This application functions to automatically apply the recipe to a series of images, either randomly collected for 2D analysis, or to comprise a 3D stack. For 3D work, additional steps such as tilt-correction, image alignment, and volume reconstructions may all be performed, permitting a single fluid workflow.

If a batch process was performed for 2D analysis, the results may be opened directly in the Processed Image Editor (see Figure 3). This environment permits accelerated review of a recipe's segmentation quality across all processed images. If necessary, manual segmentation editing is available, also in very efficient form. Finally, if either global or feature measurements are desired, they can be easily output from all processed images in spreadsheet format.

If a batch process produced either an image stack or reconstructed volume, the 3D Toolbox (see Figure 4) provides a host of tools for interacting with, editing, visualizing, and measuring these data. If a stack or reconstruction were made with alignments, the quantitative slice-to-slice translations can be viewed as scatter plots. Cropping of the image stack or reconstruction can be performed interactively. In some cases, a direct 3D segmentation can be superior to conventional slice-by-slice segmentation. In such cases, all pre-processing and segmentation would be performed within the 3D Toolbox directly on the aligned three-dimensional data. If slice-by-slice segmentation was preferred, the 3D Toolbox can still offer many noise reduction and feature separation tools - all performed directly on the 3D segmentation. More information regarding MIPAR's capabilities and availability can be found at http://MIPAR.us. 


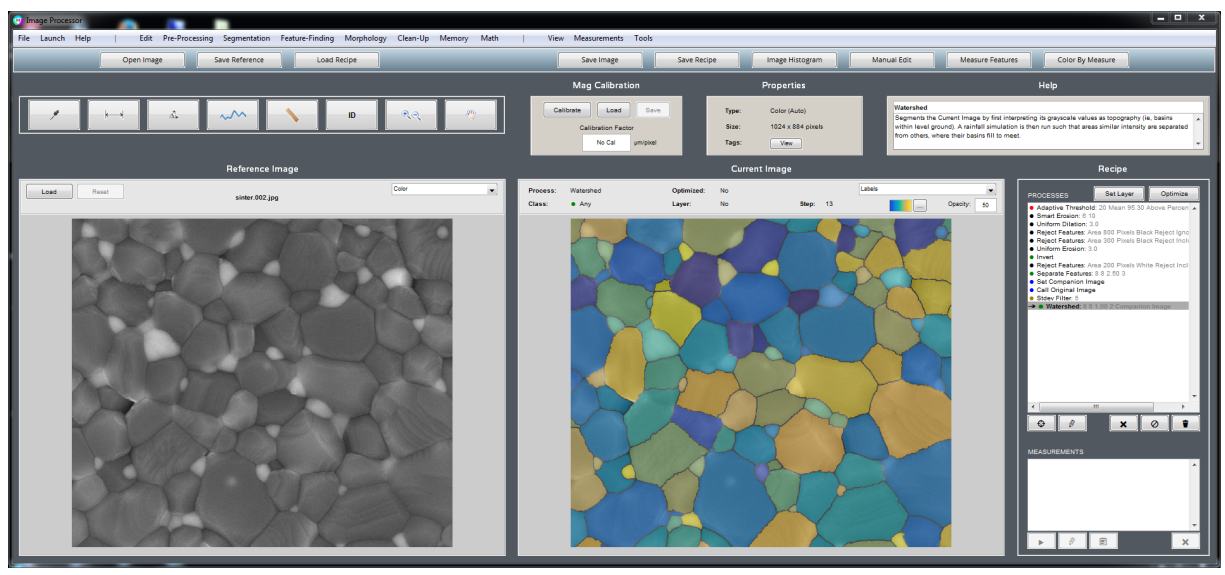

Figure 1. Screenshot of MIPAR's Image Processor application

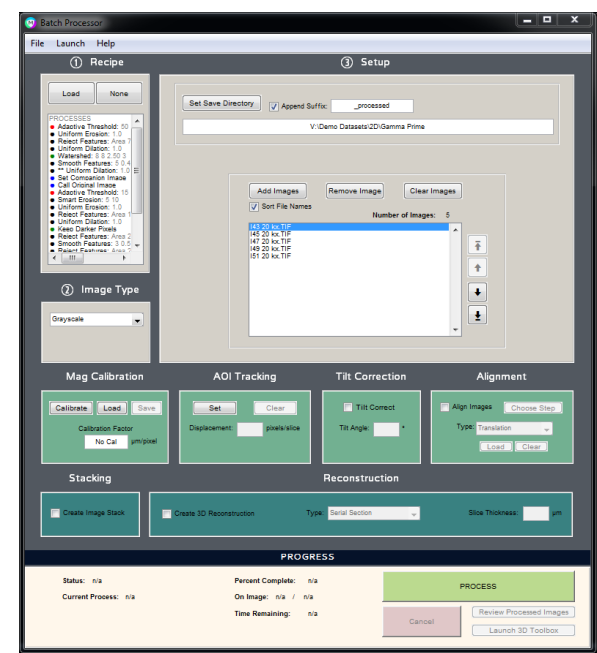

Figure 2. Screenshot of MIPAR's Batch Processor application

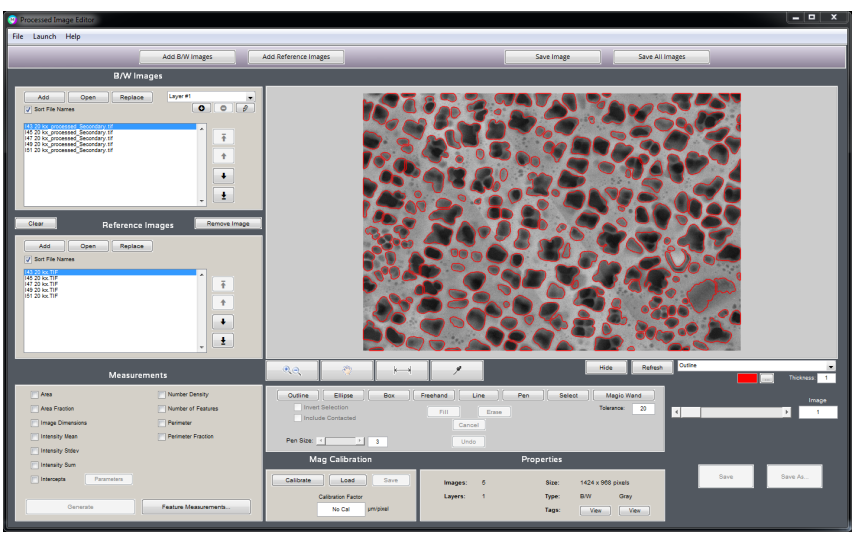

Figure 3. Screenshot of MIPAR's Processed Image Editor application

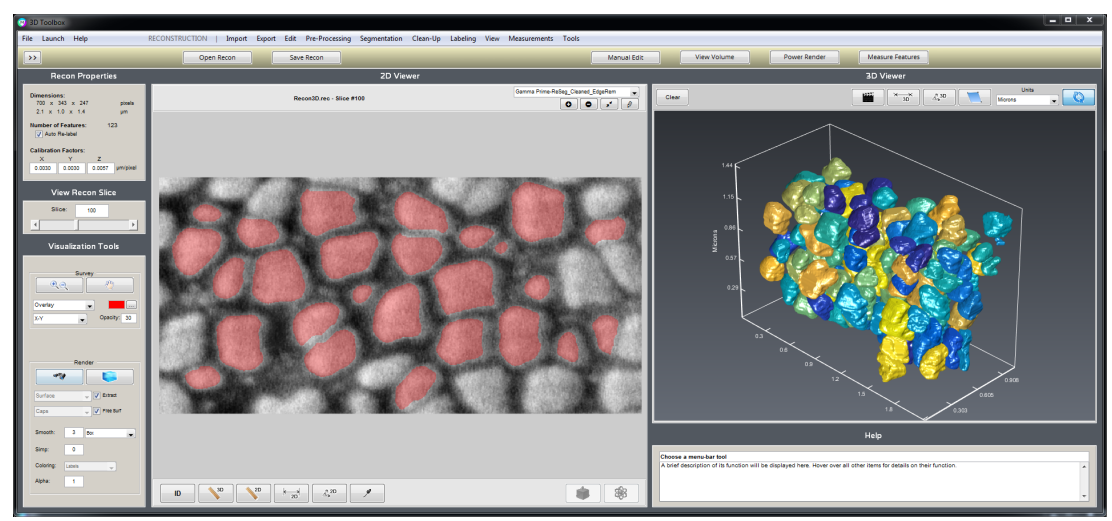

Figure 4. Screenshot of MIPAR's 3D Toolbox application 